\title{
Ethene epoxidation selectivity inhibited by twisted oxametallacycle: a DFT study on Ag surface oxide
}

\author{
Marie-Laure Bocquet and David Loffreda \\ Laboratoire de Chimie, UMR CNRS 5182, Ecole Normale Supérieure de Lyon, \\ 46 allée d'Italie, 69364 Lyon, Cedex 07, France. \\ RECEIVED DATE: 10/06/05 9:06; E-mail mbocquet@ens-lyon.fr
}

Structural figures presented here show top and lateral views of the intermediates and the transition states found along calculated regeneration pathways for the silver oxide surface catalyst: (14) the bi-reduced oxide surface left after two oxygen abstractions prior to $\mathrm{O}_{2}$ adsorption, (15) the outer molecular oxygen adsorption state called EXO (O $\alpha$ and $O \beta$ lie on-surface), (16) the inner molecular oxygen

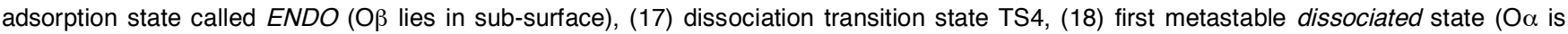
located on-surface whereas $O \beta$ and $O \gamma$ lie in sub-surface), (19) second metastable dissociated state (O $\beta$ diffuses in sub-surface plane from

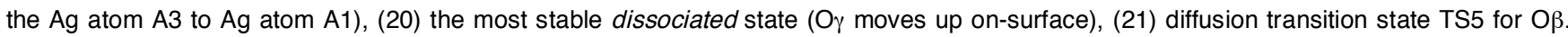
Identity numbers used refer to Fig. 3 of the main draft and Ag-O bond lengths are given in $\AA$. Clearly an additional neighboring oxygen $\mathrm{O} \gamma$ takes part to the complex regeneration mechanism.

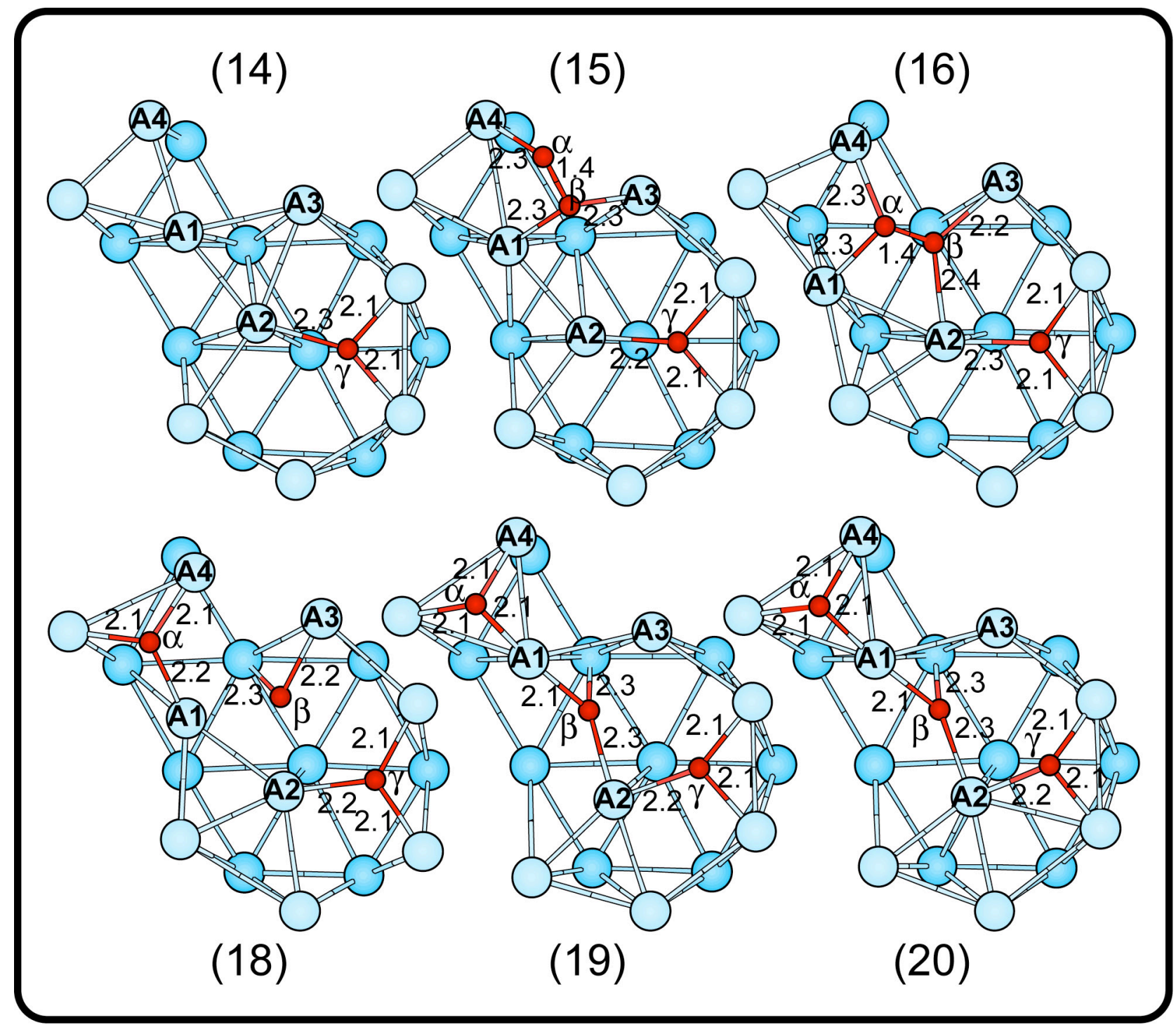




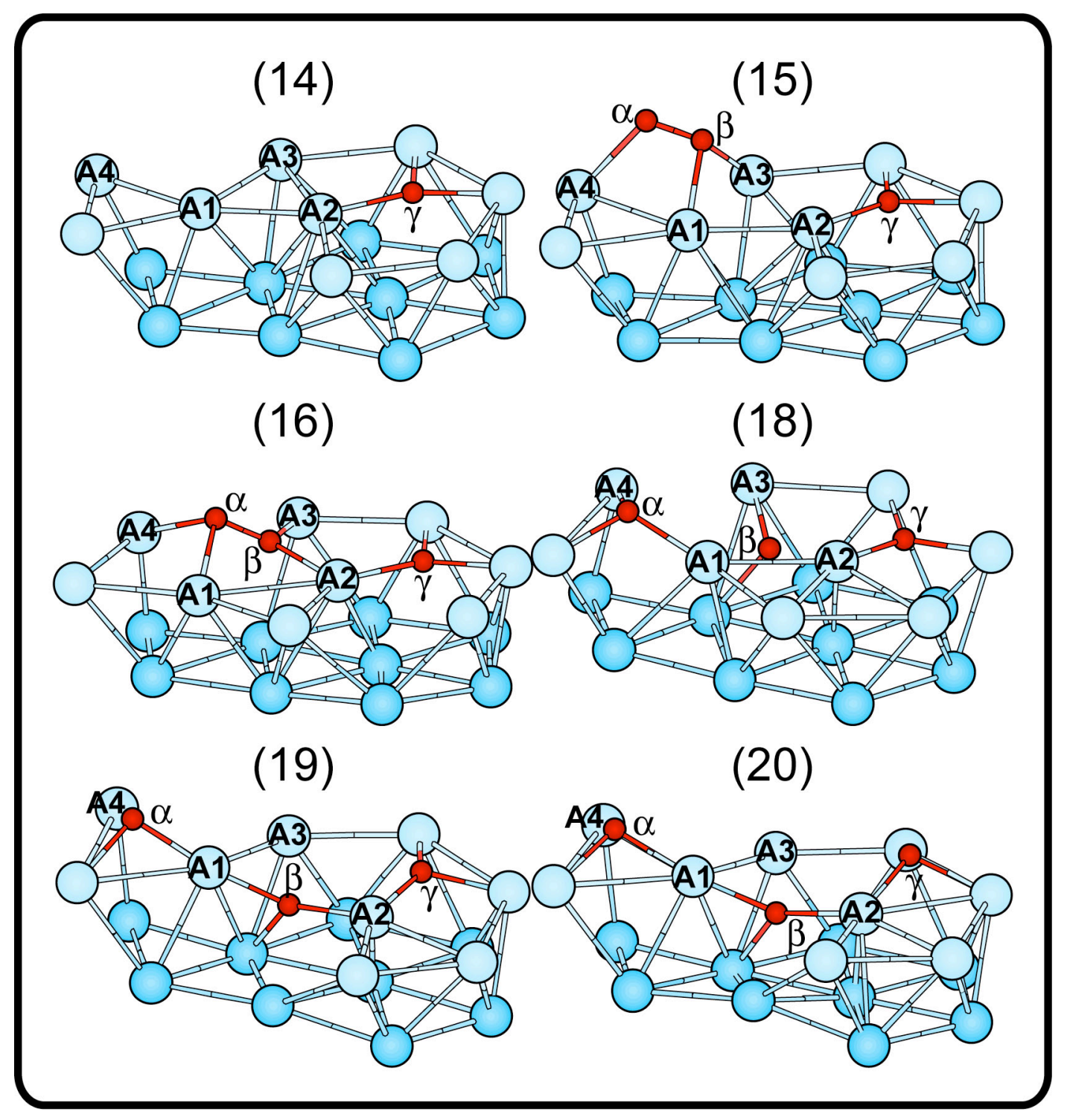




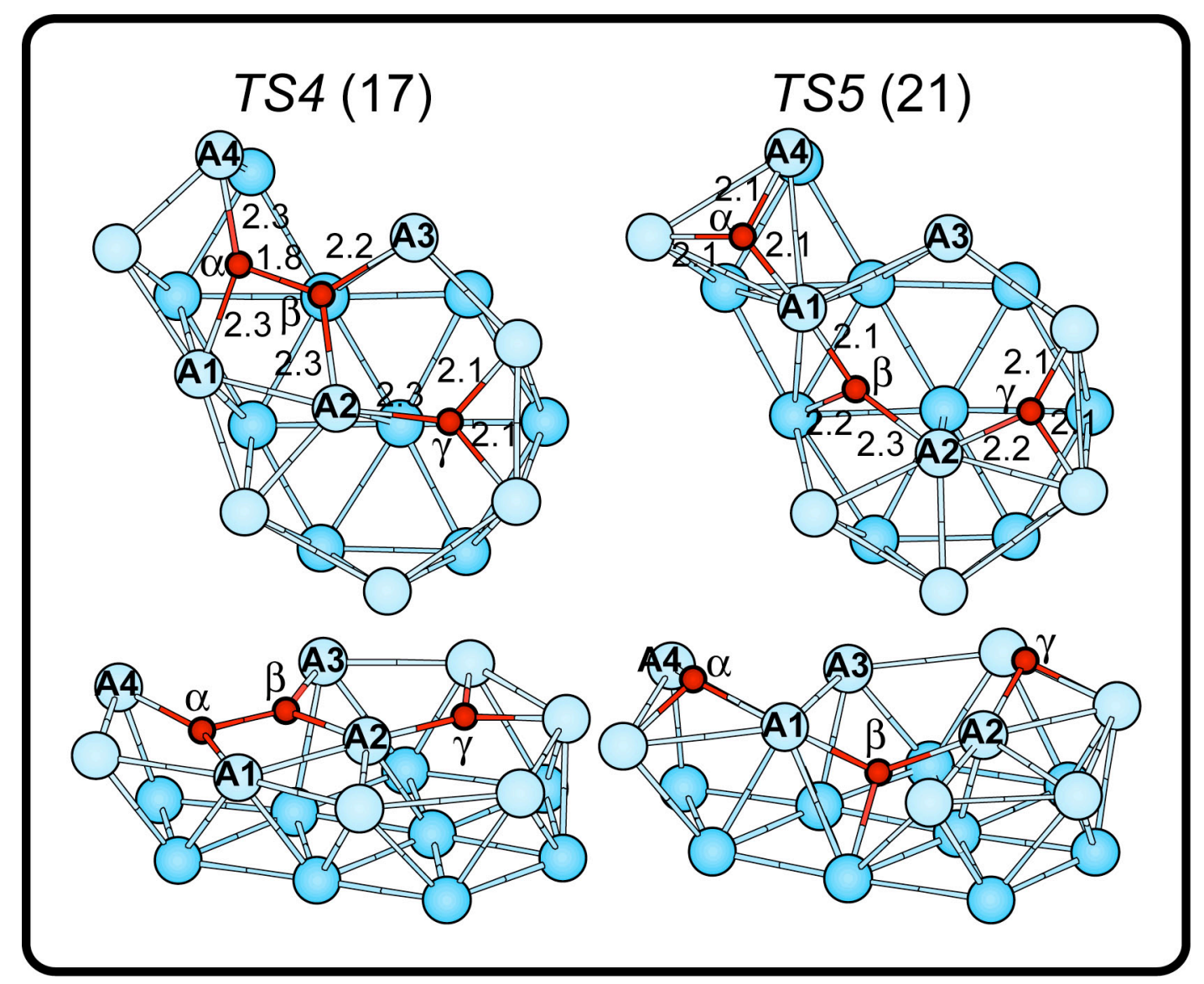

